\title{
Multidisciplinary Perspectives on Affective Experiences
}

\author{
Marianne Markowski \\ Middlesex University \\ The Burroughs \\ London NW4 4BT, UK \\ Marianne.markowski@gmail.com
}

\author{
Irida Ntalla \\ City University London \\ Northampton Square \\ London EC1V OHB, UK \\ Irida.Ntalla.1@city.ac.uk
}

\author{
Christina Kamposiori \\ University College London \\ Gower Street \\ London, WC1E 6BT, UK \\ christina.kamposiori.11
}

@ucl.ac.uk

\author{
Vasileios Routsis \\ University College London \\ Gower Street \\ London, WC1E 6BT, UK \\ v.routsis@ucl.ac.uk
}

\begin{abstract}
The paper critically reflects on the meanings and research on affective experiences in digital mediated environments. Computing, media and design, communication as well as art and cultural products are significantly engaging with aspects of affect and emotions, arguably as a part of the 'the affective turn' (Clough 2007). The text considers discussions, initiated at the AHRC funded project 'New Media, Audiences and Affective Experiences'. The project provided scope for new knowledge and skills development on researching experience and affect in digital mediated platforms and art interventions on the mentioned crossing disciplines. We regard affective experiences as the elusive factor that connects the fields and their practices, requiring attention and unfolding. The project aspired to reveal creative approaches across disciplines that 'produce and describe' affective experiences, illustrating how they synthesise and expand the notion of affect. The reflections entail a critical summary of the project's interventions, including designers and practitioners' perspectives on elaborating affective aspects in their work. Additionally, issues on multi and interdisciplinary research are considered with the paper portraying a selection of applied research methods for creating and measuring affect online and in research labs as well as the employment of design experimental approaches (see Figure 4). We conclude that some of our initial understanding of affective experiences has been assured; yet, foremost, this reflective paper highlights the complexities of creating, steering, experiencing and evaluating these types of experiences and their constant evolution with creative approaches and emerging technologies.
\end{abstract}

Affect. Experience. Art and culture. Design.

\section{INTRODUCTION}

The paper engages with the meanings of affective experiences in digital mediated environments. Interactivity and digital technologies are challenging traditional notions of reason and cognition, perception and memory, emotions and affection. This paper's focus is on the creative tensions and overlaps in the fields of design, new media, digital humanities, information communication technologies and cultural studies. It considers the 'chaotic processes that presently constitute the social' (Clough 2007: 3) situated in the selfreflexive relationship between the emergence of technological possibilities and bodily experiences. The Arts and Humanities Research Council (AHRC) funded the project 'New Media, Audiences and Affective Experiences', which initiated discussions on the challenges on understanding, conceptualising and researching digital mediated audience's experiences and their emotional and affective engagement. We have accepted that the interaction between human and computational systems has moved beyond notions of usability and user experience, arguing that the interaction is undoubtedly more complex and multiple. Technology is beyond a mere tool for the human. The intensities and encounters performed and elicited through the interaction with media present complex power dynamics involving individuals, collectives, institutions and commercial interests.

The paper is not intending to develop a unified definition of affective experiences and thereby simplifying or potentially reducing the complexities of the notion. The aims lie on exploring practices and understandings routed in empirical research examples, collaboration and discussions, subsequently reflecting on our learning, offering digestions to the research community as impulse of their interpretations of affective experiences. Our reflections do not simply juxtapose the topics and talks explored, but also entail an interdisciplinary element, leading to the following two main themes drawn out of the project. In the following text, we will discuss the two main themes, practice and 
research methods, and present empirical project examples, in order to highlight phenomena, stimulate thought and develop new questions. But before we present these we ask the question of why it is useful to research the affective aspects of these types of experiences and provide further background information on the AHRC project.

\section{WHY AFFECTIVE EXPERIENCES?}

Cultural studies have developed a rich base to think about emotions, feelings and affect, but the understanding of the fields related to digital culture is largely absent (Kuntsman 2012). At the same time, the various uses of technological innovations in the social sphere have shaken our engagement with events, histories, art and culture and brought the question of emotional and physical encounters to the foreground again. The understanding of affect is often used loosely as a synonym for emotion (Wetherell 2012) bringing more of a troublesome when we try to communicate this type of experience through the different practices. The two main connotations of affect are firstly as emotion that derives from the psychological point of view and secondly, a more general view embraced by the social sciences that highlights its element of a forceful encounter that comes prior to consciousness (Massumi, 1987). Emotions are part of the change in the relation between affect and cognition, necessary part of the interpretation, expression, evaluation of circumstances that provide information about relations to other objects and events, including cultural institutions and implicit political power structures. There are theories about affect and emotion, which come from a wide range of disciplines - e.g. philosophy, biology, psychology, anthropology and musicology.

Experience itself is a complex term by its etymology. It can be seen as immediate contact with the body and the world or observation of a happening and the event as a memory, an act where knowledge is pursued. It is an involvement, participation or engagement with exteriority resulting in interiority as an act of abstraction and judgment. The contact between the individual and the 'social', the individual and an environment leads to an exploration of emotions. Encounters with digital media technologies, online acts, digital sites are not still, but can create communities of feelings (Ferreday 2009) becoming mediators and repositories of affect (Kuntsman 2012).

Presentations from the AHRC project (see Section 2.1) introduced the experience of affect through abstract artworks that deconstruct and resist identification but carry rather a forceful, often transformative encounter. That encounter is argued to produce strong non-cognitive, corporeal processes or states of affect (Massumi, 1987). Discussions also lied on theoretical models and ideas deploying the conception of affect, introduced the idea of experience in the context of analysing texts of literature. The complexity of how affect can be deployed and exercised by the artists, curators and designers remains but the significance of the encounter between the human and the medium, is certainly agreeable. The audience is argued to become the main focus of the researcher, the designer, and the artist. Performances and artworks are open to be manipulated, felt, changed and become an individual's very personal and affective experience. Computing, design and usability have also become part of the discussion on affect with the emergence and development of affective computing, emotional usability and emotional design (Picard 2003). The emergence of new digital and ubiquitous technological interfaces altered the bodily-lived experience of people and subsequently their perceptions, leading to a constantly evolving loop of experiences, which need to be situated in a larger socio-economical and ethical context. For example, interaction design practices (Höök 2013) have produced new concepts such as the affective alarm clock (Wensveen et al. 2002) or a lamp targeting values such as social helpfulness (Ross 2008).

\subsection{Background work on the 'New Media, Audiences and Affective Experiences' project}

The AHRC funded project brought together disciplines of media arts and design, arts, digital humanities and cultural studies with an aim to comprehend how a researcher, an artist and a designer understand and produce experiences that carry emotional and affective qualities. How do we explore affective encounters in relation to interactivity, audience participation and interactive experiences? How do artists' work and engagement with audiences reveal affective qualities? What is the role of affect and emotion when researching audience's experience? How should we formulate research questions, research methods, and research dissemination? What methods researchers use and how affective encounters can be captured? To address these guiding questions we held three seminars between March and July 2013 exploring the following topics: (i) Research and digital technologies, (ii) Theoretical approaches on digital and new media research, (iii) Users and audience. We further organised the "Affective Experiences" conference on 9th December 2013 where a selected group of speakers presented their work and research. Talks were divided into the following four panels: (i) Affect in Media, Art and Design, (ii) Research online, (iii) Art evoking affect, and lastly (iv) Experiencing and measuring affective experiences. 
Providing an open space for dialogue along the diverse practices and research fields was challenging. The plurality of the definitions per se, the different disciplines and their uses of the specific concepts and the limited timeframe indicated the complexity of the themes. Nevertheless, an open discussion did occur along the participants on the different parts of the projects (seminars and conference) that showcase the necessity and motives for hybrids to emerge leading to new strands of work and knowledge exchange. In the following part, we will summarise engaging points from speakers and contributors to the project, reflecting upon the concepts presented.

The first section Practice (see Section 3) focuses on the production of affective experiences and the practices employed by artists and/ or designers in relation to audience participation and interactive experiences. Audience participation is a familiar and arguably essential feature in these practices, as it is integral to the new forms of relationship developing in the intersections of art, technology and the social. The second part Research methods (see Section 4) concentrates on the use of methods to capture affective experiences, including online and lab experiments. In the latter theme epistemological stances, the formulation of research questions and the utilisation of research methods come into focus.

\section{PRACTICE}

This section introduces practices related to the use of interactive and digital technologies that can potentially lead to the creation of novel experiences for audiences while engaging them in an emotional and affective level. Firstly, we provide an example, which illustrates how a new narrative mode based on story shapes affect the audiences' experiences. Secondly, we offer two artists' perspectives on curating experiences in kinaesthetic empathy.

Helen Bendon, artist and lecturer from Middlesex University, presented the challenges she faced when creating a locative audio drama (Scratch) in association with BBC and the investigation she conducted in regards to story shapes for the narrative as part of the associated research (Parry, Bendon et al. 2008). In order to guide the users, who were walking through the site with a GPS enabled PDA while listening to the drama, the narrative had to give clues as to where they were in the story or when they were about to leave the site. As there was not an obvious beginning, middle and end of the story or physical landmarks on the site indicating these, the overarching narrative had to allow flexibility for a person's free movement on the site. After trials with the GPS technology, BBC storywriters had to react and adjust the story elements to keep audiences immersed and 'on site'. The fragmented narratives gave room for personalised experiences to occur, allowing the audience freedom in the process of sense-making, which was based on project audio cues as well as personal mood and interests.

The new media artists Nic Sandiland, from Middlesex University London as well as, Philip Szporer and Marlene Millar, from Montreal, presented their projects, which were based on phenomenological approaches considering the bodily experience in installations. Sandiland introduced examples of his interactive art work based on video and dance (Sandiland \& Flexer 2011) in order to propose a mis-en-scene of modulation. His intentions were to blur the boundaries between the audience and the artwork, and, thus, the dynamic motions of dance. The artist wanted the audience to feel a physical experience similar to a guitar player's "experience of the vibration of the string". For this reason, he used the technique of video scrubbing employing pressure pads and a life size video projection of a video of a dancer in his installation. The effect he created was that the viewer moved the dancer by walking towards the projection and back. He describes the exchange between the artwork and the audience experiencing the installation as "modulation" (see Figure 1). According to Sandiland, it is in the modulation, in this bodily-immersed relation with the artist's intentionality through the artwork, where the viewer experiences kinaesthetic empathy.

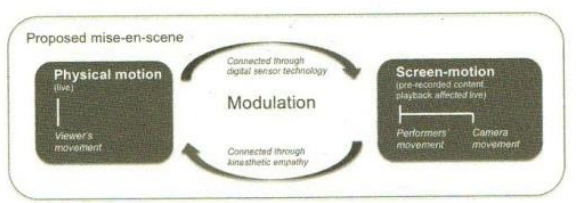

Figure 1: Sandiland's diagram of "modulation" (Sandiland 2013)

Artists Szporer and Millar discussed in their presentation a video dance piece bringing 3-D filmmaking technique as an agency of kinaesthetic empathy for live performance. The work encapsulated practical and theoretical aspects of movement, screens and spectatorship where the viewer is engaged with Chinese martial arts in a 3D landscape.

The movement of the body is certainly vital for our understanding of feeling, emotion and empathy in these artworks. The first perspective was guided by the technological format influencing the story shape and thus the spheres where users were moving to explore the story. Moving beyond task performance and communicative interactivity (e.g. feedback loops), the interest lies on experience-centred art and design practices, which employ interaction in order to trigger empathy, emotion and the broader 
notion of affect. So far the question of affect in media arts and design brought to the fore the body, action, movement, format, perspective and empathy. In the artworks described above we see technology as both a medium for producing experiences and a tool for artistic and cultural practice. The aim seems to be movement beyond spectatorship towards an affective environment where the audience is becoming part of the performance through visual and bodily engagement. Earlier notions of spectatorship have been criticised for excluding emotion, affect and feeling (Muller \& Kuhn 1993).

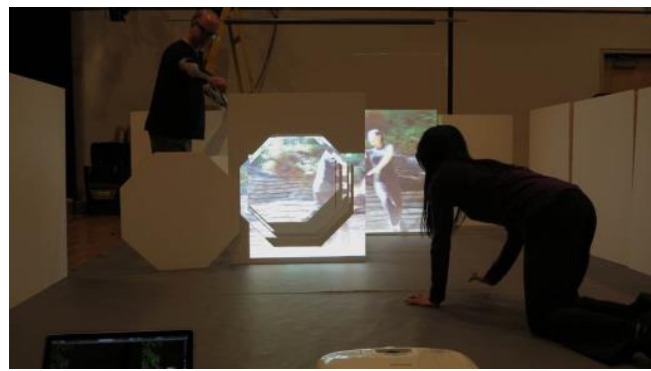

Figure 2: Marlene Millar, Philip Szporer 'Leaning On A Horse Asking For Directions'

\subsection{An experimental approach to creating affective experiences in interactions}

Dr Mie Norgaard and Lars Hoegh from Aarhus University presented their experimental approach to exploring the affective relationship between human and object. Norgaard had given Hoegh (an interaction design student) five keywords to turn a simple activity such as displaying a photo into an experience, which could alter the relationship between the human and the object (the photo in this case). Design and usability research have developed emotional awareness as well, taking into account pleasure, enjoyment and fun (Norman 2003). The five value-laden keywords were: magical, dirty, Japanese, organic and fragile interaction. Hoegh explored aesthetic qualities and affect in his designs by drawing on inspiration from dramaturgy, critical design and slow technology.

At this point, it is worthwhile mentioning the reaction of the conference audience, which responded to the experiments with murmurs, such as "Oh", "Ah", smiles and some laughter, as well as a general feeling of suspense. Thus, we believe that Hoegh, also achieved to create affective experiences through his engaging presentation to the audience on the day. Foremost, this experimental approach has to be considered in the educational context; in this case the tutor giving the design student a mundane task to create into an experience. Through this task, Hoegh was constantly challenged in being a reflective practitioner while exploring the concept of affect.
Within this educational challenge, the experimental approach appears to blur the duality of making and measuring.

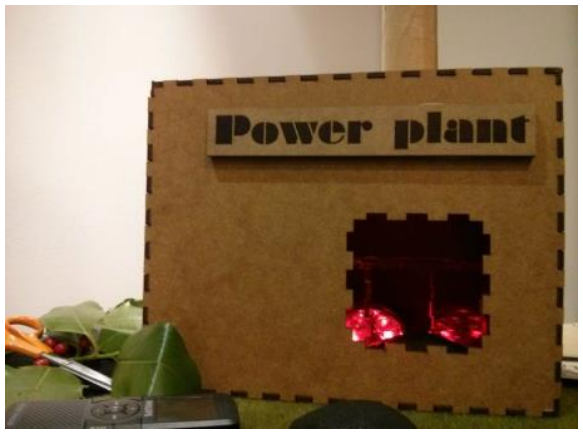

Figure 4. Hoegh's design experiment for the keyword "dirty"; the aesthetical exploration of the interaction asked users to add coals into the power plant before the photo was "produced" out of a printer

\section{RESEARCH METHODS}

The emergence of digital media have influenced new ways to capture users' and audiences' reactions, and a range of new behaviours have been developed (Back \& Puwar 2012). Questions such as the role of affect and emotion when researching audience's experience arise. How should we formulate research questions, research methods, and research dissemination? What methods researchers use and how affective encounters can be captured?

\subsection{Research performed online}

The Research Online panel strived to bring out some practical examples of the methodological techniques currently being used in relation to emotional and cultural engagement. It aimed as well as to critically assess the implications and limitations of the application of such kind of technology for research purposes. The panel consisted of three distinguished speakers from diverse academic backgrounds.

From a digital humanities perspective, Professor Melissa Terras, Director of the UCL Centre of Digital Humanities, talked about Textal (http://www.textal.org), a new and innovative text analysis mobile software developed at UCL, that can be used to visualize and conceptualise the relationships between words in online environments. Textual analysis of user-generated content across the numerous social media platforms that are available today is becoming increasingly popular in measuring affect and emotion. One of the most commonly used social media for such purposes is Twitter (Roberts et al. 2012). The museum sector has also been engaging vastly with the online environments, increasingly 
dynamic web portals and associated content, digital archives, social networks, blogs, and online games (Kidd 2014). Jenny Kidd, presented an analysis of thirty museum online games which reveal uservalue of empathetic engagement in this context leading to questions concerning empathy in relation to interaction with museum content in the game context, design and online presence. Internet sites are argued to be objects of feelings revealing emotional and empathetic intensities (Kuntsman 2012), providing a rich source for understanding and investigating audiences and users interactions. One of the limitations of these approaches is that they only consider the exchange between those who participate in the online spheres, neglecting those who are not taking part.

\subsection{Observing affective experiences}

Ethnographical approaches and observation are integral parts of researching human experiences, allowing the researcher to make sense of interactions between individuals and their environments. Digital and online technologies, as our examples also illustrate, have offered new tools and methods to artists and scholars for researching audience behaviour and experience through observation.

One of the first inputs in the seminar was the work of Dirk vom Lehn, Sociologist and Lecturer, at King's College London. Vom Lehn, Heath \& Hindmarsh (2005) have written extensively on the ways that people interact with and around particular exhibits as well as between each other, viewing the experience of museum visit emerging from the interactions with other events, groups and individuals that happen within the same space. Their research methodology involves video-based field studies where the researchers explore those interactions in details. Tom Flint from Napier University presented his research into the appropriation of interactive media by the audience. His research journey started with observing interactivity in the "Public" - a museum showcasing interactive and digital art in West Bromwich, UK. He was inspired by the observation how teenagers had ignored instructions, but developed their own playful interaction, an ad-hoc game, with a visual projection-based installation (Flint \& Turner 2012). His main argument proposes that enriching interactivity only manifests itself when audiences assign their own meanings and take ownership of the affective experiences created (Akah \& Bardzell 2010). Theano Moussouri, from UCL Institute of Archaeology, discussed the multiple ethical and practical challenges emerging from the use of modern digital and online technologies as a toolkit of audience research. In order to develop her views and analyse the idea of motivation for the audience and visitor in particular, she used a case study on the automated analysis of spatial behavior achieved by the deployment of tracking GPS technology on visitors-volunteers at the London Zoo (Moussouri \& Roussos 2011).

Various forms of observations, such as direct observation, video recorded observation or movements tracked through a digital device, offer only one way of capturing audience's feedback, and is evidently not enough to uncover the hidden affective experiences. None of the approaches presented so far can work in isolation, but have to be seen as part of a larger set of methods in order to interpret the experiences captured.

\subsection{Measuring affective experiences in a lab}

Dr. Harry Witchel and Carina Westling, University of Sussex, and their cross-disciplinary team measured the embodied responses of the users while engaging with audio-visual interactive media in a lab setting (Witchel et al. 2013). The seated volunteer participants were fully motion captured and their movements are video analysed. The research team also observed the participants. Both types of data collection were used to develop a better understanding of the nuances of affective 'body and cognitive' engagement while entrainment. So far their results indicate that there is a difference in the inhibition of non-instrumental body responses (e.g. scratching, twitching) depending on the type of stimuli. This approach, concentrating on the bodily and cognitive engagement as well as measuring the emotional engagement with a scale, tries to quantify the affective response spectrum. Overarching questions in regards to the cultural applicability of this research in different countries still remain as well as the thought of whether results could be used to manipulate audiences positively or negatively.

\section{REFLECTIONS}

Reflection occurs when you revisit a process, question and rethink situations with the aim to gain new knowledge. It is a learning process as it is a process of learning through experience. A reflective practice carries a critical view within, contributing to the personal and broader practice. In most cases with the completion of a project, the collaboration and the mental exercise of the themes examined also terminate. In our case, we viewed the interdisciplinary collaboration as an on-going process. The line between multidisciplinarity and interdisciplinarity is blurred but we understand that multidisciplinary research draws from a number of disciplines aiming to benefit a specific field whereas the latter looks at issues that may talk to various disciplines and their borderlines. From this etymology we understand that indeed the research 
is interdisciplinary when more than one discipline, theories and perhaps methods are used (Richards 1996: 124) and encapsule a multidisciplinary team. Interdisciplinary research, on the other hand, acts as a common space for developing new methods to understand problems (Youngblood 2007).

Diverse epistemologies and approaches are grasped through the different examples discussed. Artists such as Sandiland, Szporer and Millar embrace phenomenological approaches in the interpretation and understanding of their work. Phenomenology suggests that our experiencing and thinking is always subject to human constructions. It is a study of experience and how we consciously embrace sensory perceptions, imagination, thought and emotions. Furthermore, human interaction design and design research have been embracing phenomenological approaches indicating the increased interest in the significance of emotions and embodiment (McCarthy \& Wright 2004). The physical body becomes central on shaping experiences, developing understanding and interacting in a world where embodied cognition is essential to human cognition (Pecher \& Zwaan 2005). The examples demonstrated in the paper (see Section 3) seem to be agreeable on the importance of embodiment in affecting the human experience. Other examples of the paper (See Section 4.3) provide a very different interdisciplinary approach on understanding affective experiences through measurement of emotions and reactions. Drawing mostly from a scientific point of view and positivism, they analyse a felt experience by measuring the embodied responses of the users while engaging with audiovisual interactive media in a lab setting (Witchel et al. 2013). 'Productive pragmatism' (Melles 2008) has been frequently the philosophical background for product experience producing research activities in techno-scientific fields. Many research projects in interaction design and design research explore through making. Experienced practitioners apply reflection-in-action on a foremost unconscious level (Schoen 1991). The five design experiments (see Section 3.1) demonstrate how a student can be trained to be a self-reflective practitioner who constructs experiences addressing the affective states of the audiences (or the tutors initially).

A further interesting point to note about this AHRC project was that while a shift in audiences' participation was generally agreed upon, the conference call received very little input (neither in abstracts nor in full discussions) on the changing role of the audience, visitor or user. The dichotomy between creator / artist / practitioner versus audience / visitor / user still seemed to be held, despite the blurring of experience production processes and interactivity. However, a Special
Issue was conducted on 'Researching audiences in digital mediated and interactive experiences' as part of the project (Ntalla \& Vom Lehn 2014). From an overarching point of view, this project has brought out and confirmed when looking at affective experiences the focus is on the relational nature between body, cognition, mood, perspective, intentionality and aesthetics.

Finally, we decided to move the collaboration further through the organisation of another event. For the purpose of stirring constructive discussions around the issue of affect between the different disciplines, our team will use the format of the workshop (participatory) as an experiment. Thus, in our participatory workshop we invite scholars to bring with them their own research, meaning their projects, methods and perspectives of affect. The ultimate goal is twofold; firstly, to identify and understand empirical issues in researching affective experiences as they emerge during the workshop and, secondly, to create a network of researchers who are interested in the topic, currently non-existent, while providing a platform for constructive exchange.

\section{CONCLUSIONS}

To sum up, this paper provides rather an exploratory discussion on interpreting and researching affective experiences. The project did not provide concrete results or specific answers to the initial questions; its purpose was not to present definitions on fluid and multidimensional concepts like affective experiences. Instead our aspirations were to creatively expand concepts and bring attention to the complexity of the questions asked, whilst assuring some of our initial understandings.

Designers and media artists are more and more concerned with audience's body, their actions and movements challenging their perspectives, allowing felt and empathic experiences to emerge. Researchers are utilizing traditional methods and experimenting with new multidisciplinary approaches to comprehend audience's interaction with various media and cultural practices online and onsite. Indeed dichotomies such as mind-body, social-natural, human-nonhuman, cognitiveaffective are questioned. The experience of the digital era today is increasingly moving beyond forms of representation, involving the corporeality of perception, with artists, practitioners and designers exploring the many intersections between affect, emotion, sensation and action.

\section{Acknowledgements}

The project is a collaboration of doctoral students from City University London, Kings College 
London, Middlesex University, New London Graduate School (NLGS) and University College London in the academic fields of Creative Industries and Practice, Art and Design and Digital Humanities.

\section{REFERENCES}

Akah, B. \& Bardzell, S. (2010) Empowering Products: Personal Identity through the Act of Appropriation, 4021-4026.

Back, L. \& Puwar, N. (eds.) (2012) Live Methods, Wiley-Blackwell.

\section{Clough, P. \& Halley J. (eds) (2007) 'The Affective} Turn: Theorizing the Social', Durham: Duke UP.

Ferreday, D. (2009) Online Belongings: Fantasy, Virtuality, Community, Peter Lang, Oxford.

Flint, T. \& Turner, P. (2012) The Role ofAppropriation in the Design of Engaging Artefacts.

Höök, K. (2013) Affective Computing. In R. F. Soegaard, Mads and Dam (ed.), The Encyclopedia of Human-Computer Interaction (2nd ed.). The Interaction Design Foundation.

Klauser, K. \& Walker, V. (2007) It 's About Time: An Affective and Desirable Alarm Clock, (August), 22-25.

Kuntsman A. (2012) Digital Cultures and the Politics of Emotion: Feelings, Affect and Technological Change, Palgrave Macmillan, Basingstoke and New York.

Massumi, B. (1987) Notes on the Translation and Acknowledgements." In Gilles Deleuze and Felix Guattari, A Thousand Plateaus. Minneapolis: University of Minnesota Press

McCarthy, J. \& Wright, P. C. (2004) Technology as experience., MA: MIT Press, Cambridge.

Melles, G. (2008) New Pragmatism and the Vocabulary and Metaphors of Scholarly Design Research. Design Issues, 24(4),

Moussouri, T. \& Roussos, G. (2011) Automated Analysis of Spatial Behavior at the London Zoo. In 24th Annual Visitor Studies Association Conference ' Sustaining a Community of Learners', 24-27 July 2011, Chicago, Illinois.

Muller, M. \& Kuhn, S. (1993) Special issue on Participatory Design. Communications of the ACM, 36(4).

Ntalla I. (2013) Engaging audiences on on-going social debates through interactive and immersive exhibits. The International Journal of Inclusive Museum, 105-116, ISSN: 1835-2014 (print).
Ntalla, I. \& Vom Lehn, D. (2014) Researching audiences in digital mediated and interactive experiences: Special Section Introduction. Participation: Journal of Audience and Reception Studies, 11(1), 213-215.

Parry, N., Bendon, H., Boyd Davis, S., \& Magnus, M. (2008) Locating drama: a demonstration of location-aware audio drama. In International conference on interactive digital storytelling (pp. 41-43).

Pecher, D. \& Zwaan, R. A. (eds.) (2005) Grounding Cognition: The Role of Perception and Action in Memory, Language and Thinking. ed. Cambridge University Press: Cambridge, UK.

Picard, R. W. (2000) Toward computers that recognize and respond to user emotion. IBM Systems Journal, 39, 705-719. Retrieved November 1, 2006, from Academic Search Premiere electronic database.

Richards, D. G. (1996) The meaning and relevance of 'synthesis' in interdisciplinary studies. The Journal of Education, 45(2), 114-128.

Roberts, K., Roach, M., Johnson, J, Guthrie, J., \& Harabagiu, S. (2012) EmpaTweet: Annotating and Detecting Emotions on Twitter. Proceedings of the LREC (pp. 3806-3813).

Ross, P. R. (2008) Ethics and aesthetics inintelligent product and system design. Technical University, Eindhoven, The Netherlands.

Sandiland, N. (2013) Modulation in Interactive Video Installation. In I. Ntalla, M. Markowski, C. Kamposiori, \& V. Routsis (eds.), Affectives Experiences (p. 14). London, UK.

Sandiland, N., \& Flexer, Y. (2011) Points of View (2011) Flexer \& Sandiland. Retrieved from http://flexerandsandiland.com/archives/nicsandiland/installations/points-of-view/

Schoen, D. A. (1991) The Reflective Practitioner: How Professionals Think in Action. Ashgate Publishing Limited.

vom Lehn, D., Heath, C., \& Hindmarsh, J. (2005) Examining exhibits: Interaction in museums and galleries. Communication \& Cognition, 38(3-4), 229-247.

Wensveen, S., Overbeeke, K., \& Djajadiningrat, T. (2002) Push me, shove me and I show you how you feel. Proceedings of the Conference on Designing Interactive Systems Processes, Practices, Methods, and Techniques - DIS '02, 335. doi:10.1145/778757.77875

Wetherell, M. (2012) Affect and emotion: A new social science understanding'. Thousand Oaks, CA: Sage, London. 
Witchel, H., Westling, C., Tee, J., Healy,A.,Needham, R., \& Nachiappan, C. (2013) Mean head and shoulder heights when seated: subconscious postural cycles during discrete computerised stimuli. In Conference on cognitive ergonomics.
Youngblood, D. (2007) Interdisciplinary Studies and the Bridging Discipline: A Matter of Process. Journal of Research Practice, 3(2). 